\title{
A Model of Temperature-Dependent Young's Modulus for Ultrahigh Temperature Ceramics
}

\author{
Weiguo Li, ${ }^{1}$ Ruzhuan Wang, ${ }^{1}$ Dingyu Li, ${ }^{1}$ and Daining Fang ${ }^{2}$ \\ ${ }^{1}$ College of Resource and Environmental Science, Chongqing University, Chongqing 400030, China \\ ${ }^{2}$ LTCS and College of Engineering, Peking University, Beijing 100871, China \\ Correspondence should be addressed to Weiguo Li, wgli@cqu.edu.cn
}

Received 27 September 2010; Accepted 2 January 2011

Academic Editor: Harold Zandvliet

Copyright ( 2011 Weiguo Li et al. This is an open access article distributed under the Creative Commons Attribution License, which permits unrestricted use, distribution, and reproduction in any medium, provided the original work is properly cited.

Based on the different sensitivities of material properties to temperature between ultrahigh temperature ceramics (UHTCs) and traditional ceramics, the original empirical formula of temperature-dependent Young's modulus of ceramic materials is unable to describe the temperature dependence of Young's modulus of UHTCs which are used as thermal protection materials. In this paper, a characterization applied to Young's modulus of UHTC materials under high temperature which is revised from the original empirical formula is established. The applicable temperature range of the characterization extends to the higher temperature zone. This study will provide a basis for the characterization for strength and fracture toughness of UHTC materials and provide theoretical bases and technical reserves for the UHTC materials' design and application in the field of spacecraft.

\section{Introduction}

Ultrahigh temperature ceramics (UHTCs) is a family of materials that have melting points higher than $3000^{\circ} \mathrm{C}$ and can be potentially used at temperatures above $2000^{\circ} \mathrm{C}$ in an oxidizing environment $[1,2]$. So they are possible aircraft thermal protection materials that can meet the requirements of thermal protection of hypersonic aircraft. The hypersonic aircraft is an advanced and strategic technology in the field of aerospace. With their sharp nose-cones and leading edges, as well as extended exposure to the atmosphere during long flights, these aircrafts suffer from serious aerodynamic heating. This heating severely challenges the materials used for thermal protection and structures, especially given the harsh demand for the reliability of materials under the mechanical/heat coupling conditions. However, currently, due to lack of sufficient knowledge on UHTC thermal protection materials, development and application of these materials have been limited.

The UHTC materials are quite different with the traditional ceramic materials, as their mechanical behavior and failure mechanism under a long time of dynamic thermal/mechanical coupling loads are very different with material properties at room temperature, and their relevant material parameters are quite sensitive to temperature. Although some studies involved in the effects of components, manufacturing process, microstructure, density and environmental temperature of UHTC materials on strength, and fracture toughness have achieved series of promising research results, the research temperature is far not to reach the actual level. The current research focuses mainly on experimental research, but the experimental system is more decentralized, the theoretical analysis is not systematic, some temperature-dependent empirical formulas are no longer applicable, and the basis of establishment of the original theory no longer fully meets the requirement under high temperature (above $2000^{\circ} \mathrm{C}$ ). In this paper, based on the original empirical formula, by considering that the UHTCs used as thermal protection materials must meet the precise design requirements, the study focuses on method for the characterization of temperature-dependent Young's modulus for UHTC materials under high temperature. The study will provide a basis for characterization for thermodynamic properties of UHTC materials. 


\section{The Effect of Temperature on Young's Modulus}

The study of effect of temperature on Young's modulus is extremely important for the strength under high temperature and thermal shock properties of ceramics and refractories. When the temperature increases, the atomic thermal vibrations increase, and this will cause the changes of lattice potential energy and curvature of the potential energy curve, so the Young's modulus will also change. And with the increase of temperature, the material will have a volume expansion. Thus we know that the change of Young's modulus with temperature involves two aspects; one is the atomic binding force, and the other is the volume of material. Therefore, the relationship between Young's modulus and temperature is relatively complicated. However, a linear dependence has been observed for Young' modulus of several refractory oxides including aluminum oxide, magnesium oxide, and thorium oxide above room temperature. Wachtman et al. considered that the linear dependence at high temperature is probably characteristic of such a high degree of excitation of the vibrational modes, and based on the requirement of the third law of thermodynamics that the derivative of any elastic constant with respect to temperature must approach zero as the temperature approaches absolute zero, he proposed an empirical formula of temperaturedependent Young's modulus [3]

$$
E=E_{0}-B T \exp \left(-\frac{T_{m}}{T}\right)
$$

where $E_{0}$ is the Young's modulus as the temperature is $0 \mathrm{~K}, T$ is the absolute temperature. $B$ is the parameter depending on the material, which is the value of slope of the Young's modulus-temperature curve decreasing from a constant value as the temperature is lowed. It is said to be related to Gruneisen constant $\gamma$. The $\exp \left(-T_{M} / T\right)$ is a single Boltzmann factor, and $T_{M}$ is the parameter depending on the material which is suggested to have a correlation with the Debye temperature $\Theta$. And $\gamma$ and $\Theta$ are related to the volume thermal expansion and the specific heat, respectively.

Opeka et al. obtained temperature curves of Young's modulus from the literature [4], as shown in Figure 1. We can see the variation law of Young's modulus with temperature in Figure 1 that firstly it is similar to the linear decrease, while the temperature reaches a certain value the decline rate increases rapidly, and then a gradual slowdown followed. So there are two turning points on the curve in which we generally consider that the deformation mechanism changes. Therefore, if we want to descript the variation law well so as to fully reflect the changes of material's deformation mechanism at different temperature stages, the empirical formula built must be able to reflect the laws at different change trend stages. However, from the mathematical properties of formula (1) we can know that it only can be used to describe the linear stage and the stage of accelerated reduction under low temperature, yet it cannot well describe the phenomenon of the decline rate slowing down under high temperature.

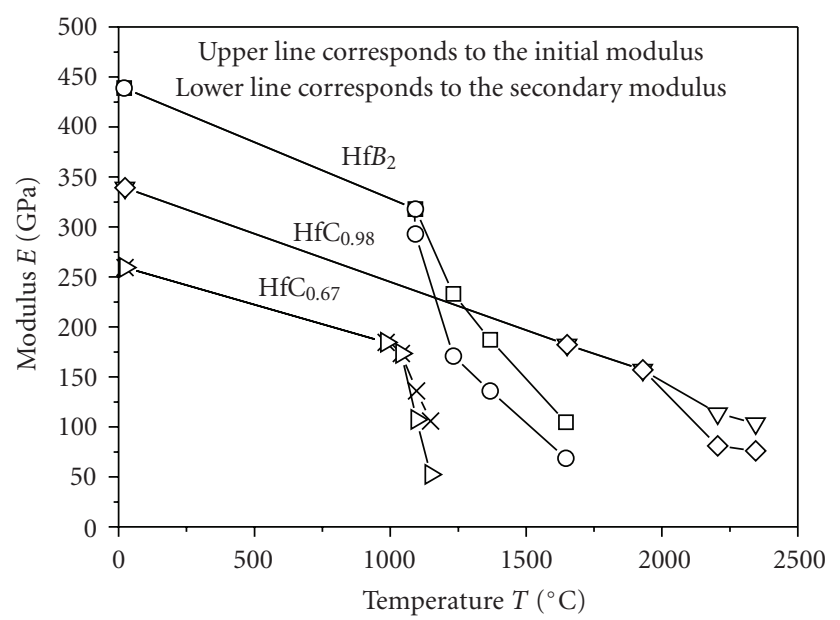

FIgURE 1: Temperature curves of Young's modulus (bending test) [4].

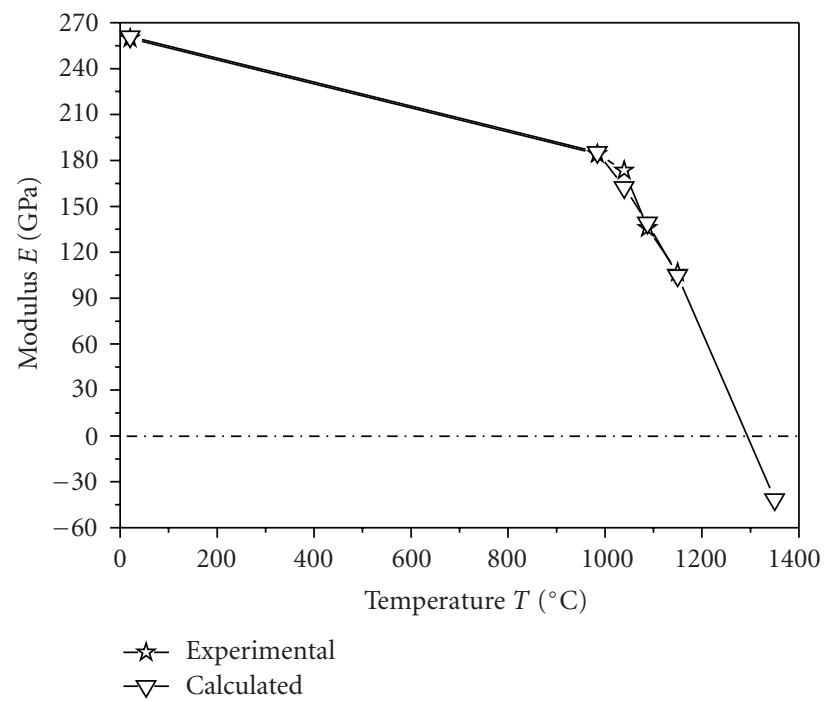

Figure 2: Temperature curves of Young's modulus $\left(\mathrm{HfC}_{0.67} T_{m}=\right.$ $\left.3890^{\circ} \mathrm{C}\right)$.

Figure 2 is experimental temperature curve of Young's modulus of $\mathrm{HfC}_{0.67}$ material and calculated curve of Young's modulus of $\mathrm{HfC}_{0.67}$ material which is simulated from the original formula (1). In the simulation, $T_{m}$ is taken to be $3890^{\circ} \mathrm{C}$, and $B$ is taken to be 4 . As can be seen from Figure 2, the original empirical formula (1) can well simulate the experimental curve of $\mathrm{HfC}_{0.67}$ material the in literature [4]. But with the further increase of temperature, the value of Young's modulus decreases rapidly, which reduces to zero or even becomes negative as the temperature is $1300^{\circ} \mathrm{C}$ or so. This obviously does not match the truth that the melting point of $\mathrm{HfC}_{0.67}$ material is up to $3890^{\circ} \mathrm{C}$. This also shows that the original empirical formula applies only at certain temperature on low temperature phase, it no longer applies at certain temperature on the higher temperature zone (generally $T$ is higher than $0.5 T_{m}$ ). So we revise formula (1) based on the analysis of experimental data of 


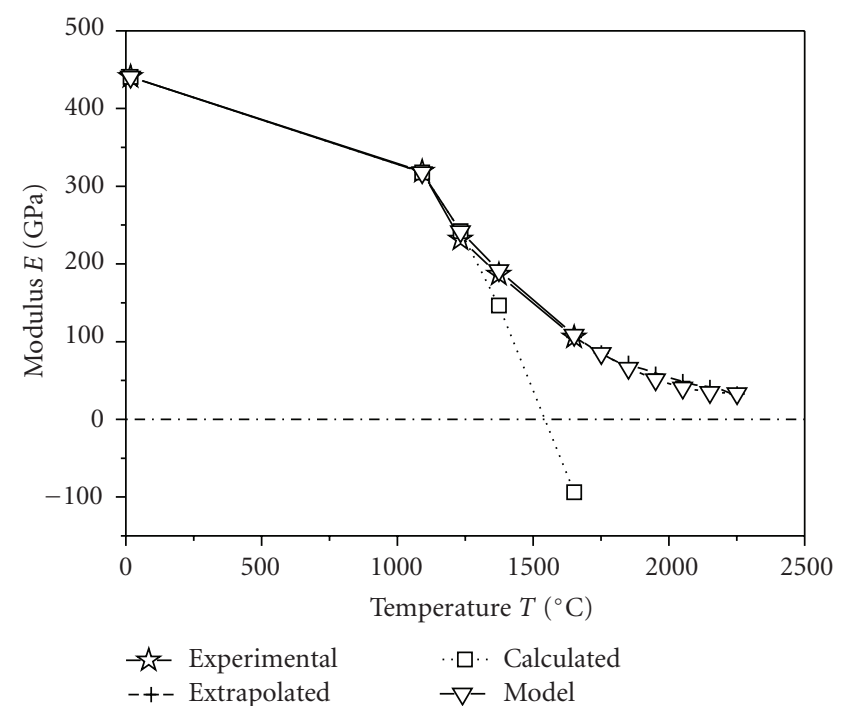

Figure 3: Temperature curves of Young's modulus $\left(\mathrm{Hf}_{2} T_{m}=\right.$ $\left.3400^{\circ} \mathrm{C}\right)$.

Young's modulus of UHTC materials and then obtain a new characterization as follows:

$$
E=E_{0}-B T e^{-T_{m} / T}+B_{1}\left(T-B_{2} T_{m}+\left|T-B_{2} T_{m}\right|\right) e^{-T_{m} / T},
$$

where $B_{1}$ and $B_{2}$ are parameters depending on the material. $B_{2}$ can be obtained from the temperature of turning point where the decline rate of experimental data of Young's modulus begins to slow down.

As we lack the experimental data under high temperature, we make appropriate extrapolations on the basis of original data, as shown in Figure 3. And we simulate those extrapolated data by using the modified formula (2), in which $T_{m}$ is $3400^{\circ} \mathrm{C}, B$ is $2.18, B_{1}$ is $0.25, B_{2}$ is 0.404 , and $B_{3}$ is 0.00058 . As can be seen from Figure 3 , the modified formula (2) can well simulate the temperature dependence of Young's modulus under high temperature. We can also see from Figure 3 that the value of Young's modulus obtained from the original empirical formula (1) becomes 0 when temperature is about $0.5 T_{m}$. The situation does not match reality. This also shows that the original empirical formula applies only at certain temperature on low temperature phase.

\section{Conclusions}

Based on the different sensitivities of material properties of UHTCs and traditional ceramics to temperature and the complexity of service environment of thermal protection system on the spacecraft, the original temperature-dependent Young's modulus model of ceramic materials is unable to describe the temperature dependence of Young's modulus of UHTC materials in their entire operating process. In this paper, a characterization applied to Young's modulus of UHTC materials under high temperature which is revised from the original empirical formula is established. The applicable temperature range of the characterization increases significantly compared to the original empirical formula and parameters which are easy to be determined. This study will provide a basis for characterization for thermodynamic properties of UHTC materials.

\section{Acknowledgment}

The authors are grateful for support from the National Natural Science Foundation of China under Grants nos. 10702035 and 90916009.

\section{References}

[1] M. Gasch, D. Ellerby, E. Irby, S. Beckman, M. Gusman, and S. Johnson, "Processing, properties and arc jet oxidation of hafnium diboride/silicon carbide ultra high temperature ceramics," Journal of Materials Science, vol. 39, no. 19, pp. 59255937, 2004.

[2] E. Wuchina, M. Opeka, S. Causey et al., "Designing for ultrahigh-temperature applications: the mechanical and thermal properties of $\mathrm{HfB}_{2}$, $\mathrm{HfCx}, \mathrm{HfNx}$ and $\alpha \mathrm{Hf}(\mathrm{N})$," Journal of Materials Science, vol. 39, no. 19, pp. 5939-5949, 2004.

[3] J. B. Wachtman Jr., W. E. Tefft, D. G. Lam, and C. S. Apstein, "Exponential temperature dependence of Young's modulus for several oxides," Physical Review, vol. 122, no. 6, pp. 1754-1759, 1961.

[4] M. M. Opeka, I. G. Talmy, E. J. Wuchina, J. A. Zaykoski, and S. J. Causey, "Mechanical, thermal, and oxidation properties of refractory hafnium and zirconium compounds," Journal of the European Ceramic Society, vol. 19, no. 13-14, pp. 2405-2414, 1999. 

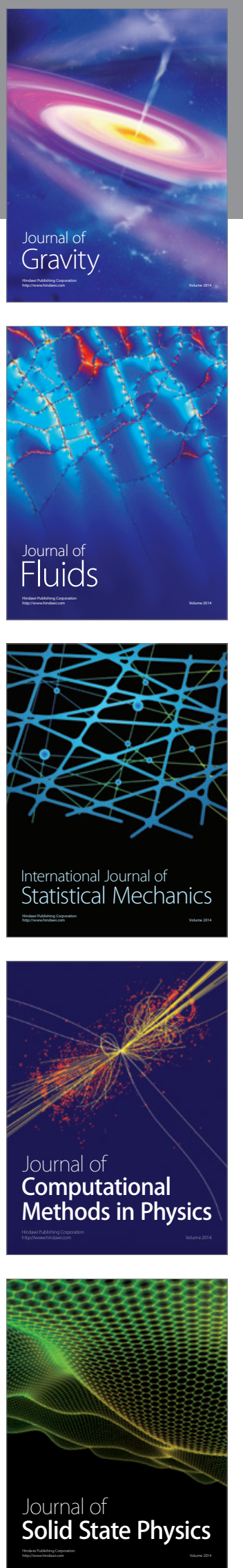

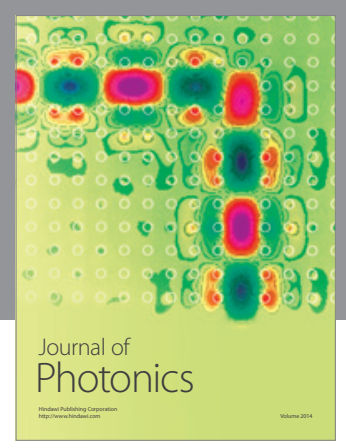

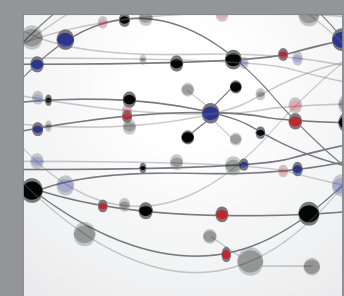

The Scientific World Journal
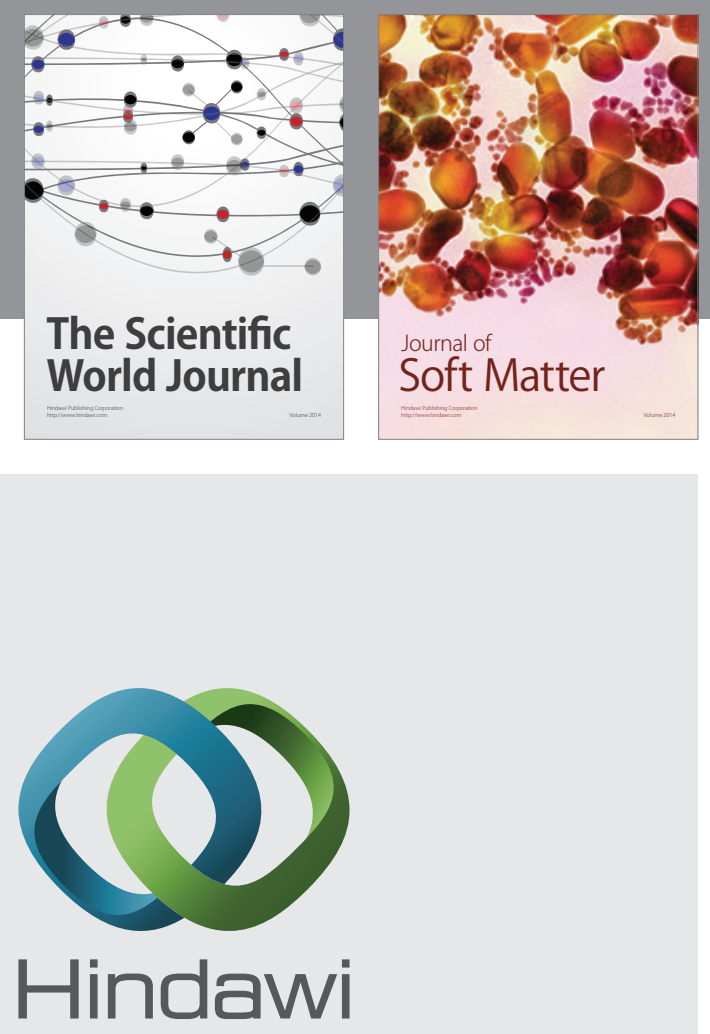

Submit your manuscripts at

http://www.hindawi.com
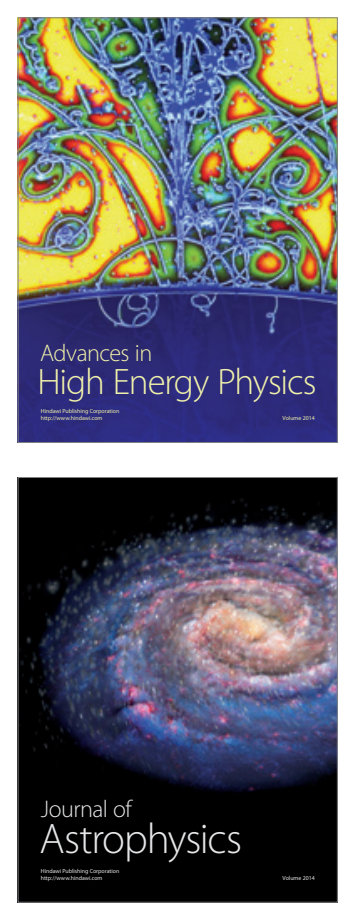
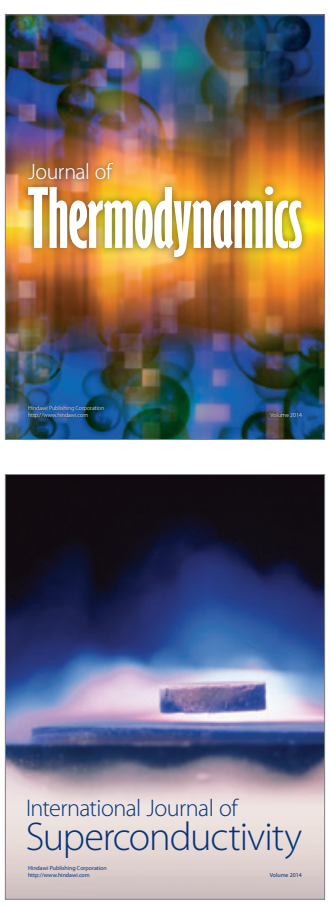
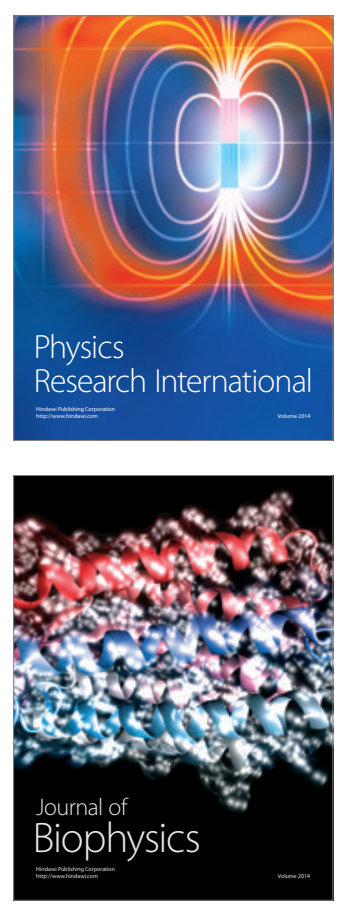
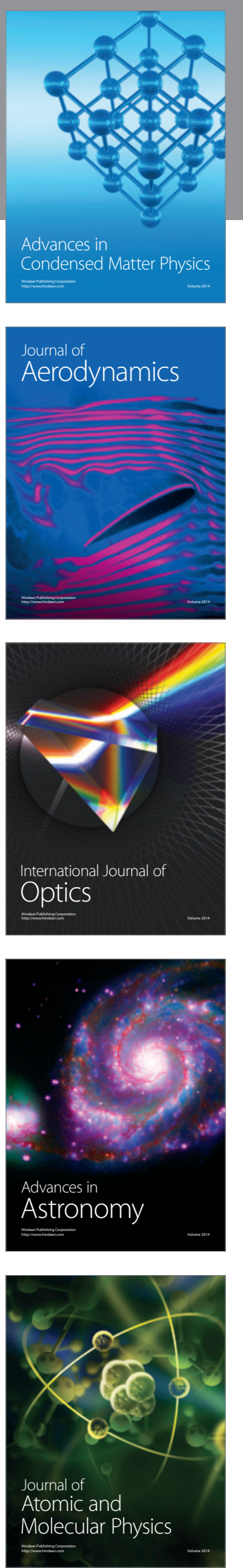